\title{
Extracting Noise Elements while Preserving Edges in Spatial Domain
}

\author{
Jalil Bushra, Fauvet Eric, and Laligant Olivier \\ Le2i Laboratory, Universit de Bourgogne, 12 Rue de la Fonderie, Le Creusot, France \\ bushra.jalil@u-bourgogne.fr
}

\begin{abstract}
In this paper, we are interested in preserving the sharp transitions and edges present inside the image. Image denoising by means of wavelet transforms has been an active research topic for many years. In this work, we used Lipschitz exponents based on wavelet transform to performs edge preservation by identifying these transitions. The smoothing part was performed by using some heuristic approach utilizing data samples and smoothness criteria in spatial domain with out prior modeling of either the image or noise statistics. The method tries to find the the best compromise between the data and the smoothing criteria based on the type of the transition present. The method has been compared with the shrinkage approach, Wiener filter and Non Local- means algorithm as well. Experimental results showed that the proposed method gives better signal to noise ratio as compared to the previously proposed denoising solutions.
\end{abstract}

Keywords: Denoising, Edge detection, Lipschitz exponent, Mean square error, Signal Smoothness.

\section{Introduction}

One of the main problem faced in the field of image processing is that of image denoising, where the goal is to obtain an estimate of the original image from an image that has been contaminated by noise. The two main limitations in any image accuracy are categorized as blur and noise and the main objective of any filtering method is to effectively suppress the noise elements. Not only that, it is of extreme importance to preserve and enhance the edges at the same time. In image processing, the region of abrupt changes contains the most of the useful information about the nature of the image. The region or the points where these changes occurred are termed as an edge. It is possible to study the nature of any edge in terms of singularity or regularity. The singularity is considered to be an important character of an edge, as it refers to the level of discontinuity or interruption present inside the image and the main purpose of the detection of such singular point is to identify the existence, location and size of those singularities. Several methods have been proposed in the past to attain these objectives and to recover the original (noise free) image. Most of these techniques uses averaging filter e.g. the Gaussian smoothing model has been used by Gabor 
[1, some of these techniques uses anisotropic filtering [23] and the neighborhood filtering [45], some works in frequency domain e.g Wiener filters [4]. In the past few years, wavelet transform has also been used as a significant tool to denoise the signal 6 678 . A brief survey of some of these approaches is given by Buades et al 9 .

Traditionally, linear models have been used to extract the noise elements e.g. Gaussian filter as they are computationally less expensive. However, in most of the cases the linear models are not able to preserve sharp edges which are later recognized as a discontinuities in the image. On the other hand, nonlinear models can effectively handle this task (preserve edges) but more often, non linear model are computationally expensive. In the present work, we attempt to propose a non linear model with the very less computational cost to restore image from noisy data samples. The method utilizes data samples and find the best compromise between the data samples and smoothness criteria which ultimately result in giving the denoise signal at a very low computational cost. we have also presented the comparative analysis of the present technique with some of the previously proposed method.

The principle of the proposed technique is given in section 2. Section 3 presents the singular points extraction method. Section 4 explains the overview of restoration method. Comparative analysis is given in section 5 and finally section 6 conclude the work.

\section{Principle of the Method}

We assume that the given data specify the model:

$$
y_{i j}=f\left(x_{i j}\right)+\epsilon_{i j} \quad \text { where } i, j=1, \ldots, n
$$

$f$ is the noise free signal, uniformly sampled (e.g., an image) and $\epsilon_{i j}$ is the white gaussian noise $N\left(0, \sigma^{2}\right)$. In the present work, the given data will always be an $n \times n$ matrix with $n=2^{N}$. The aim of the current work is to estimate the function $F:\left(f\left(x_{i j}\right)\right)_{i, j=1}^{n}$ with respect to an estimator $\hat{F}$ such that:

$$
S N R(d B)=-10 \log _{10} \frac{\sum_{i, j=1}^{n}\left(\hat{F}_{i, j}-F_{i, j}\right)^{2}}{\sum_{i, j=1}^{n} F_{i, j}^{2}}
$$

In order to estimate the function $F$, the method utilizes the data samples and performs non linear functioning to estimate the best fit. The filtering has been performed on each row and column matrix individually and at the final stage by utilizing filtering in $x$ and $y$ directions yield in fully denoised image.

$$
G=\frac{1}{\sqrt{2}} \sqrt{\left(G_{x}\right)^{2}+\left(G_{y}\right)^{2}}
$$

where $\frac{1}{\sqrt{2}}$ is the normalizing factor, $G_{x}$ is the filtered image in horizontal direction and $G_{y}$ is the filtered image in vertical direction. 

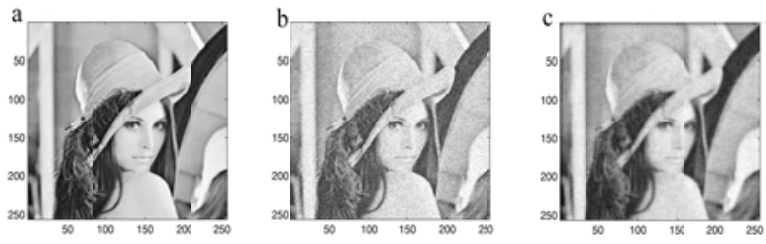

Fig. 1. a) Original Lena image, b) Lena image with "white Gaussian noise" SNR of $15 \mathrm{~dB}, \mathrm{c})$ Denoised Lena image with proposed Mse-Smooth method

\section{Estimation of Singular Points}

In this section, the Modulus maxima approach has been applied on signal to identify different signatures in the signal. Mallat proposed a method to compute the singularity of the signal by finding the local maxima. This singularity can be defined in terms of an interval or as a point wise. If the signal has constant behavior in some given interval $[\mathrm{a}, \mathrm{b}]$ then the singularity is defined in terms of interval. In our work, we focused in computing the singularities at individual points [10].

\subsection{Continuous Wavelet Transform}

The Morlet-Grossmann definition of the continuous wavelet transform for a 1dimensional signal $f(x)$, the space of all square integrable functions, is [10]:

$$
W T(a, b)=\frac{1}{\sqrt{a}} \int_{-\infty}^{+\infty} f(x) \mathrm{y}^{*}\left[\frac{(x-b)}{a}\right] d x
$$

Where $\mathrm{WT}(\mathrm{a}, \mathrm{b})$ is the wavelet coefficient of the function $f(x)$, $\mathrm{x}$ is the analyzing wavelet, $\mathrm{a}(>0)$ is the scale parameter and $\mathrm{b}$ is the position parameter.

The constant $\frac{1}{\sqrt{a}}$ is used to normalise or conserve the energy before and after the transform. It has been explained in that if the wavelet used is the first derivative of a smoothing function, then the wavelet transform $W_{1} f(s, x)$ or $W_{2} f(s, x)$ is proportional to the first (or the second) derivative of $f(x)$ smoothed by the function wavelet function.

\subsection{Lipschitz Exponent}

The singularity of the points can be described by computing their Lipschitz exponent. The Lipschitz exponent is a generalized measure of the differentiability of a function

Definition: Supposes $\mathrm{n}$ is an integer, $n<\alpha<n+1$, the signal $f(x)$ has Lipschitz a at $x_{o}$,if and only if there exists a constant $\mathrm{A}$ and $x_{0}>0$ which, that for the polynomial $P_{n}(x)$ of n-order, satisfy [10,11,12]:

$$
f(x)-p_{n}(x) \leq A\left|x-x_{0}\right|^{\alpha}
$$



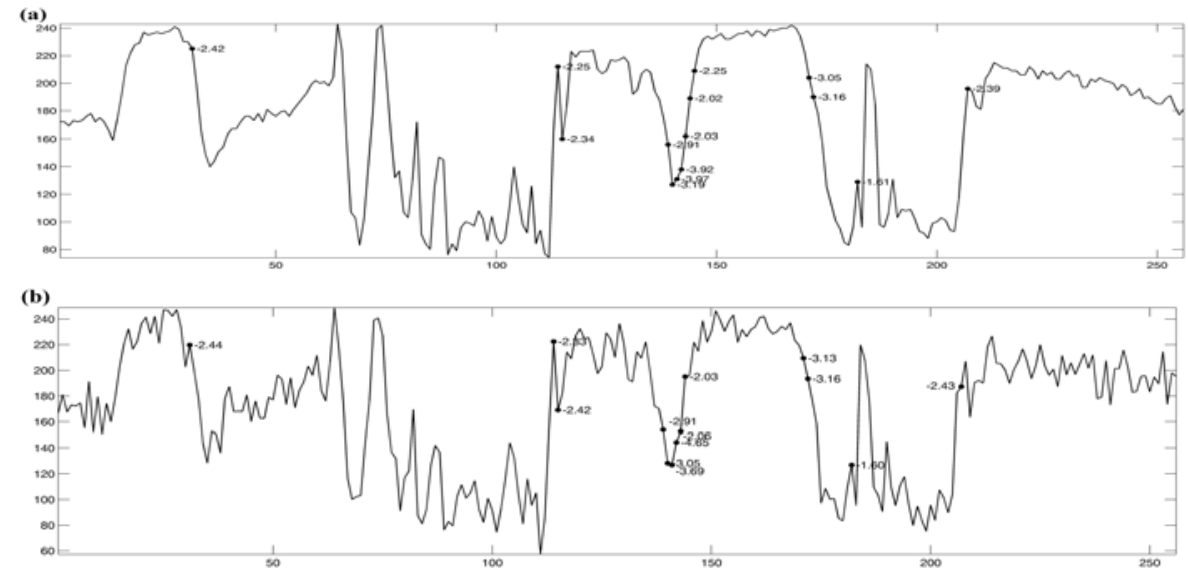

Fig. 2. a) Row No. 125 of Original Lena image, b) Row No. 125 of Noisy Lena image. Modulus maxima are marked as a circle in both images and the corresponding Lipschitz value is written in numerical form.

Where the least upper bound of $\alpha$ at the point of $x_{0}$ is defined as the regularity of $f(x)$ at the point of $x_{0}$. These Lipschitz exponent can be estimated form the slope of logarithm of modulus maxima lines across logarithm of scale by using above expression. Fig 2. shows the Lipschitz analysis of a single row of original and Noisy Lena image.

\section{Reconstruction}

The present method proposes restoring by taking into account extracted regular data samples based on their respective Lipschitz exponents as shown in Fig. 3 . The method utilizes all sampled points and smoothness (Mse-Smooth) of the signal to estimate the best fit by working in an iterative mode. The method is design for one dimensional signal, therefore it performs non linear filtering on image initially row by row and then column by column. At the final stage, by using expression in given eq.3 merging of filtering operation in horizontal and vertical direction results in giving the fully denoised image.

Lets define any one dimensional signal as:

$$
y^{k+1}=y^{k}+\lambda^{k}\left(-\frac{\partial C^{M S E, k}}{\partial y}\right)+\gamma^{k}\left(-\frac{\partial C^{M S O, k}}{\partial y}\right)
$$

$y$ denote the one dimensional signal with $l \epsilon 1, \ldots 2^{N}$ samples. $k$ define the iteration step. $C^{M S E}$ is the mean square error estimation of the restored signal with the original signal $\left(y_{o}\right)$ such that :

$$
C^{M S E}=\sum_{l=1}^{N}\left(y_{l}^{k}-y_{o}^{k}\right)^{2}
$$




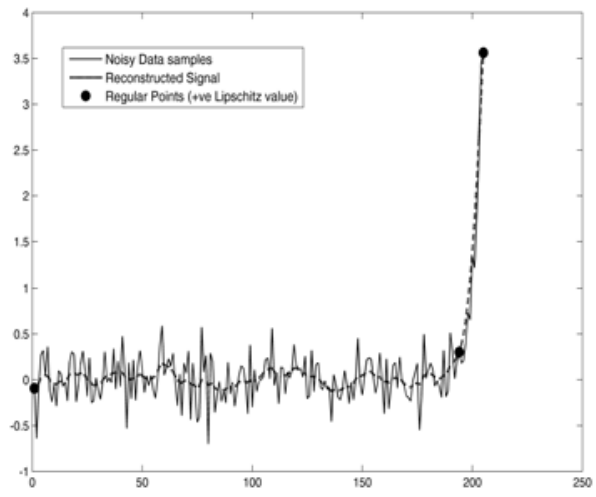

Fig. 3. Signal showing regular points (Lipschitz value $\alpha>1$ ) marked as circle. The reconstruction method preserve these regular points. Solid Line $=$ Noisy data samples, Dotted lines $=$ Reconstructed signal.

$C^{M S O}$ define the smoothness of the reconstructed subset signal.

$$
C^{M S O}=\sum_{l=1}^{N} y_{l}^{k^{\prime 2}}
$$

In order to find the value of $\lambda^{k}$ and $\gamma^{k}$ in eq.6, consider the Taylor series expansion and for the given series, in order to find the minimum mean square error we want $f^{\prime}(x+d x)=0$ therefore by simplifying, $d x=-\frac{f^{\prime}(x)}{\frac{d^{2} f}{d x^{2}}}$. we know that: $x^{k+1}=x^{k}+\lambda d x$. As $x \rightarrow Y^{k+1} \quad$ and $f \rightarrow C^{M S E, k}$ therefore by replacing the variables in $e q$ we have :

$$
y^{k+1}=y^{k}+\frac{-C^{M S E, k^{\prime}}(y)}{\frac{d^{2} C^{M S E, k}}{d y^{k^{2}}}}
$$

hence, $\lambda^{k}=\frac{d^{2} C^{M S E, k}}{d y^{k^{2}}}$ and by simplifying:

$$
y^{k+1}=y^{k}+\lambda^{k}\left(-\frac{\partial C^{M S E}}{\partial y}\right)
$$

Similarly by using the Taylor series in term of smoothing criteria, $\gamma^{k}$ comes out to be:

$$
\gamma^{k}=\frac{d^{2} C^{M S O, k}}{d y^{k^{2}}}
$$

By using eq.6, each row wise and column wise vector were restored individually and result in giving two $n \times n$ matrices, $G_{x}$ and $G_{y}$ respectively. At the last stage, by using mathematical expression given in $e q .3$, the final denoised image has been restored. 


\section{$5 \quad$ Results and Discussion}

The test image use in this work is Lena $256 \times 256$ picture. We generated noisy data from clean image by adding pseudorandom numbers (white gaussian noise) resulting in signal to noise ration (SNR) of approximately 15dB (SNR is defined in eq.2). Fig.1 shows the corrupted Lena image with white Gaussian noise (15db) and the denoising result with the proposed technique. It can be seen from the figure that, the new method denoised the image reasonably well while keeping the edges preserved. In order to illustrate the amount of the noise in the data and the effects of the denoising, we show in Fig.4, a single row (100) of the image (15dB white Gaussian noise), plotted as a curve. It can be seen from the figure that the method not only smooth the noisy part of the signal but also preserve the sharp edges or transitions to good extent. At the same time, the proposed

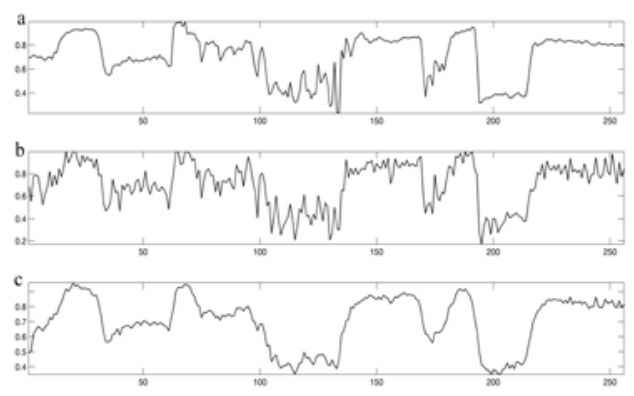

Fig. 4. a) Row 100 of original Lena image, b) Row 100 of noisy Lena image with "white Gaussian noise" SNR of $15 \mathrm{~dB}, \mathrm{c})$ Row 100 of denoised Lena image with proposed MseSmooth method
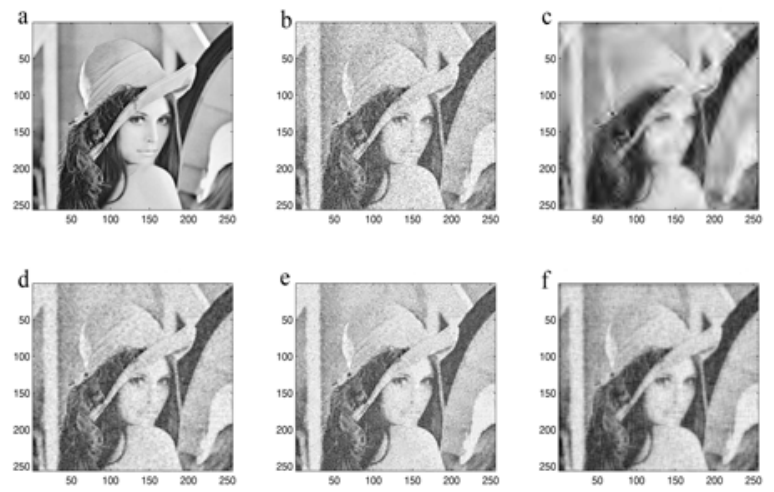

Fig. 5. a) Original Lena image, b) Lena image with "white Gaussian noise" SNR of $15 \mathrm{~dB}, \mathrm{c})$ Denoised Lena image with Visu shrink method, d) Denoising with Wiener filtering, e) Denoising with Non Local means, f) Denoising with proposed Mse-Smooth method 
method performs the whole operations at a very low computational cost which makes it useful for many real time applications (maximum time taken for any experiment was approx $16 \mathrm{sec}$ ).

\subsection{Comparative Analysis}

The human eye is able to decide if the quality of the image has been improved by the denoising method. Fig.5 displays the comparative analysis of the proposed method with other smoothing filters including Non-Local means [13, Wiener filter [4] and classical Visu shrink method [7]. The experiment has been simulated by adding white gaussian noise. It can be seen from the figure that the Visu shrink result in giving good smoothness but at the cost of the edges. Not a single edge has been preserved, this however is not, in the case of NL-means or Wiener filtering. NL-means and Wiener filtering preserved the edges reasonably well, but in this case the noise elements are visible (clearly visible on the background of Lena) and can be seen with the naked eye as well. The proposed (Mse-Smooth) method gave the best compromise of the two (blur and noise artifacts) as shown in Fig.5. Statistical results in terms of SNR $(\mathrm{dB})$ on Lena Image with different types of noise on different method is given Table 1 .

Table 1. Statistical results in terms of SNR $(\mathrm{dB})$ of "Lena Image" with different types of noise (M/S is proposed Mean-Smooth method)

\begin{tabular}{|c|c|c|c|c|c|}
\hline Noise Type & Input & NLM & Weiner & Shrink & M/S \\
\hline \hline Gaussian & 16.02 & 18.12 & 20.32 & 17.14 & 22.56 \\
\hline Speckle & 17.20 & 18.1 & 23.46 & 21.03 & 21.82 \\
\hline Salt/Pepper & 14.34 & 16.13 & 17.02 & 17.32 & 19.23 \\
\hline
\end{tabular}

\section{Conclusion}

In this work, we have presented a new denoising algorithm, based on the actual noisy image samples. Unlike other existing techniques, we have not considered modeling of an image or noise characteristics in developing the approach. Instead we have estimated the best fit of the signal by utilizing actual noisy data samples and smoothness criteria. At the same time, the proposed non linear image filtering technique works equally well in the presence of signal dependent nature of multiplicative noise in spatial domain. The proposed non linear expression have effectively directed the algorithm in the smoothing operation at a very low computational cost. For images, it is important that edges data should be preserved. The denoising algorithm presented in this work, to a large extent has satisfied the constraint that phase should not be corrupted. The effectiveness of this technique encourages the possibility of improving this approach to preserve the edges to more extent. 


\section{References}

1. Bruckstein, A., Lindenbaum, M., Fischer, M.: On gabor contribution to image enhancement. pattern recognition. Computer Methods and Programs in Biomedicine 27(1), 1-8 (1994)

2. Malik, J., Perona, P.: Scale space and edge detection using anisotropic diffusion. IEEE Transactions on Pattern Analysis, 629-639 (March 1990)

3. Morel, J.M., Alvarez, L., Lions, P.L.: Image selective smoothing and edge detection by nonlinear diffusion. Journal of Numerical Analysis 29, 845-866 (1992)

4. Yaroslavsky, L.: Digital picture processing - an introduction. Springer, Heidelberg (1985)

5. Manduchi, R., Tomasi, C.: Bilateral filtering for gray and color images. In: Proceedings of the Sixth International Conference on Computer Vision, pp. 839-846 (1998)

6. Donoho, D., Coifman, R.: Translation-invariant de-noising. In: Wavelets and Statistics, pp. 125-150. Springer, Heidelberg (1995)

7. Donoho, D.: De-noising by soft-thresholding. IEEE Transactions on Information Theory 41, 613-627 (1995)

8. Alecu, A., Munteanu, A., Tessens, L., Pizurica, A.: Context adaptive image denoising through modeling of curvelet domain statistics. Journal of Electronic Imaging 17, 033021-17 (2008)

9. Morel, J., Buades, A., Coll, B.: On image denoising methods, Technical Report CMLA (2004)

10. Mallat, S.: A wavelet Tour of Signal Processing. Academic Press, London (1998)

11. Laligant., O., Jalil, B., Fauvet, E.: Noise and artifacts removal utilizing significant features. In: Signal Processing Symposium, Poland, June 8-10 (2011)

12. Laligant., O., Jalil, B., Fauvet, E.: Piecewise image denoising utilizing discontinuous edges. In: The Eleventh International Conference on Pattern Recognition and Information Processing, Minsk, Belarus, May 18-20

13. Morel, J.M., Buades, A., Coll, B.: A non-local algorithm for image denoising. In: IEEE Computer Society Conference on Computer Vision and Pattern Recognition, pp. 60-65 (2005) 